\title{
Macrolides sensitize EGFR-TKI-induced non-apoptotic cell death via blocking autophagy flux in pancreatic cancer cell lines
}

\author{
SHUNTARO MUKAI $^{1}$, SHOTA MORIYA ${ }^{2}$, MASAKI HIRAMOTO $^{2}$, HIROMI KAZAMA $^{2}$, \\ HIROKO KOKUBA $^{3}$, XIAO-FANG CHE ${ }^{2}$, TOMOHISA YOKOYAMA ${ }^{4}$, SATOSHI SAKAMOTO ${ }^{6}$, \\ AKIHIRO SUGAWARA ${ }^{7}$, TOSHIAKI SUNAZUKA ${ }^{7}$, SATOSHI OMMURA $^{7}$, \\ HIROSHI HANDA ${ }^{5}$, TAKAO ITOI ${ }^{1}$ and KEISUKE MIYAZAWA ${ }^{2}$
}

\begin{abstract}
Departments of ${ }^{1}$ Gastroenterology and Hepatology and ${ }^{2}$ Biochemistry, ${ }^{3}$ Laboratory of Electron Microscopy, Departments of ${ }^{4}$ Clinical Oncology and ${ }^{5}$ Nanoparticle Translational Research, Tokyo Medical University, Tokyo; ${ }^{6}$ Department of Biological Information, Graduate School of Bioscience and Biotechnology, Tokyo Institute of Technology, Yokohama; ${ }^{7}$ Kitasato Institute for Life Sciences and Graduate School of Infection Control Sciences, Kitasato University, Tokyo, Japan
\end{abstract}

Received September 3, 2015; Accepted October 15, 2015

DOI: 10.3892/ijo.2015.3237

\begin{abstract}
Pancreatic cancer is one of the most difficult types of cancer to treat because of its high mortality rate due to chemotherapy resistance. We previously reported that combined treatment with gefitinib (GEF) and clarithromycin (CAM) results in enhanced cytotoxicity of GEF along with endoplasmic reticulum (ER) stress loading in non-small cell lung cancer cell lines. An epidermal growth factor receptor tyrosine kinase inhibitor (EGFR-TKI) such as GEF induces autophagy in a pro-survival role, whereas CAM inhibits autophagy flux in various cell lines. Pronounced GEF-induced cytotoxicity therefore appears to depend on the efficacy of autophagy inhibition. In the present study, we compared the effect on autophagy inhibition among such macrolides as CAM, azithromycin (AZM), and EM900, a novel 12-membered non-antibiotic macrolide. We then assessed the enhanced GEF-induced cytotoxic effect on pancreatic cancer cell lines BxPC-3 and PANC-1. Autophagy flux analysis indicated that AZM is the most effective autophagy inhibitor of the three macrolides. CAM exhibits an inhibitory effect but less than AZM and EM900. Notably, the enhancing effect of GEF-induced cytotoxicity by combining macrolides correlated well with their efficient autophagy inhibition. However, this pronounced cytotoxicity was not due to upregulation of apoptosis induction, but was at least partially mediated through necroptosis. Our data suggest the possibility of using macrolides as 'chemosensitizers' for EGFR-TKI therapy in
\end{abstract}

Correspondence to: Professor Keisuke Miyazawa, Department of Biochemistry, Tokyo Medical University, 6-1-1 Shinjuku, Shinjuku-ku, Tokyo 160-8402, Japan

E-mail: miyazawa@tokyo-med.ac.jp

Key words: pancreatic cancer, autophagy, EGFR, macrolides, gefitinib, ER stress, necroptosis pancreatic cancer patients to enhance non-apoptotic tumor cell death induction.

\section{Introduction}

Pancreatic cancer is one of the most difficult types of cancer and is a major cause of cancer-related deaths worldwide, because only $20 \%$ of patients have resectable-stage cancer at the time of diagnosis. In addition, pancreatic cancer is largely resistant to systemic chemotherapy $(1,2)$. In patients with metastatic pancreatic cancer, the 5-year survival rate is only $2 \%$. Recently, novel molecular agents targeting specific biologic pathways that are activated and involved in the pathogenesis of cancer have been developed to treat various types of cancer. Since the epidermal growth factor receptor (EGFR) is overexpressed in many pancreatic cancers, EGFR-targeting therapy appears promising (3). In a recent randomized phase III clinical trial, erlotinib, an EGFR tyrosine kinase inhibitor (EGFR-TKI) in combination with gemcitabine demonstrated improvement in survival compared to gemcitabine monotherapy (median overall survival, 6.24 vs. 5.91 months; $\mathrm{P}=0.038$ ) (4). Therefore, erlotinib in combination with gemcitabine has been approved as a major chemotherapy drug for advanced pancreatic cancer, based on high-level evidence $(5,6)$. However, the median overall survival rate for these patients indicates extremely poor prognosis compared to that of other types of cancers. Thus, more effective therapeutic strategies are required to improve therapeutic outcome.

Macroautophagy (hereafter, autophagy) is evolutionally well-conserved in eukaryotes as the major degradation mechanism whereby cellular proteins and organelle are sequestered into a double-membrane vesicle (autophagosome) and are delivered to lysosomes. They are then membrane-fused (autolysosome) and cargos are degraded for metabolic recycling by lysosomal hydrolytic enzymes, including protease $(7,8)$. It has become apparent that many aggressive cancer cells have upregulated autophagy and are dependent on autophagy for survival (9). EGFR tyrosine kinase (EGFR-TK) is overex- 
pressed or somatic mutationally activated in a broad range of human cancers, including NSCLC and pancreatic cancers (10). Activated EGFR suppresses autophagy via activation of mammalian targets of rapamycin (mTORs), as well as tyrosine phosphorylation of Beclin-1 (11-13). In response to ligand binding, EGFR becomes a dimer and is activated via tyrosine phosphorylation. Phosphorylated EGFR subsequently activates intracellular pro-survival and anti-apoptotic signals. One of the downstream activated EGFR signals involves the $\mathrm{PI} 3 \mathrm{~K} / \mathrm{AKT} / \mathrm{mTOR}$ pathway, made up of well-established negative regulators for autophagy (14). In response to nutrient and growth factor availability, mTOR promotes cell growth while suppressing autophagy. In addition, the activated EGFR binds the autophagy initiating protein Beclin-1, leading to multisite tyrosine phosphorylation and enhanced binding to autophagy inhibitors, including Rubicon (15). Thus, exposure to EGFRTKIs such as gefitinib (GEF) and erlotinib (ERL) induces autophagy via cancellation of the PI3K/AKT/mTOR pathway, as well as blocking phosphorylation of Beclin-1, which subsequently leads to dissociation of Rubicon from Beclin-1 to initiating autophagy $(13,15)$. A recent report confirmed that inactivated endosomal, but not cell surface EGFR interacts with LAPTM4B, resulting in a complex formation for recruiting Rubicon from Beclin-1 (16). As described above, dissociation of Rubicon from Beclin-1 is essential for autophagy initiation. Cancer cells with higher EGFR expression could thus be supported and survive by efficient autophagy induction under various metabolic stresses, such as low nutrient and hypoxic conditions due to insufficient vascularization $(15,16)$. Therefore, if autophagy induction in response to EGFR-TKI promotes tumor cell survival, combined treatment with an autophagy inhibitor may promote tumor cell death.

We and others have reported that macrolide antibiotics such as clarithromycin (CAM) and azithromycin (AZM) inhibit autophagy flux (17-19). We also reported that combined treatment with the proteasome inhibitor bortezomib and CAM for simultaneous blocking of the ubiquitin-proteasome system and the autophagy-lysosome system resulted in pronounced apoptosis induction of myeloma cells along with accumulation of intracellular ubiquitinated proteins and aggresome, which results in pronounced endoplasmic reticulum (ER) stress loading (20). Under accumulation of unfolded proteins inside the ER lumen, a series of adaptive responses occur: i) attenuation of translation for repression of unfolded protein accumulation; ii) induction of chaperon proteins for proper refolding; and iii) exporting of unfolded proteins to outside ER, following their polyubiquitination and degradation by proteasome [ER-associated degradation (ERAD)]. However, when ER stress loading exceeds these adaptive capacities, apoptosis is induced via transcriptional activation of a proapoptotic transcription factor such as C/EBP homologous protein (CHOP)/GADD153 and the other mechanisms $(21,22)$. Many lines of evidence now confirm that apoptosis induction via ER stress loading in cancer cells is a novel potential therapeutic strategy (23).

We recently reported that combined treatment using GEF and CAM resulted in pronounced cytotoxicity as well as ER stress loading in NSCLC (24). We also reported that macrolide antibiotics, including CAM and AZM, enhanced bortezomib-induced cytotoxicity in multiple myeloma cells (19). As EGFR-TKI induced cytoprotective autophagy in NSCLC, we hypothesized that the efficiency of macrolides in blocking autophagy might directly affect the enhanced cytotoxicity in refractory pancreatic cancer cells. In the present study, we used a novel 12-membered EM900, which shows anti-inflammatory and/or immunomodulatory effects, without possessing antibacterial activity (25), as well as CAM and AZM to compare their effects of: i) enhancing GEF-induced cytotoxicity; ii) blocking autophagy flux; and iii) ER stress loading in pancreatic cancer cell lines. Our data clearly exhibited a positive correlation between blocking efficiency in autophagy inhibition and pronounced EGFRTKI-induced cytotoxicity. This result suggests the possibility of using a macrolide in combination with EGFR-TKI therapy for pancreatic cancer.

\section{Materials and methods}

Reagents. GEF and erlotinib hydrochloride (ERL) were purchased from Biomolecules (Hamburg, Germany), and gemcitabine hydrochloride was purchased from Tokyo Chemical Industry Co.,Ltd. (Tokyo, Japan). They were dissolved in dimethyl sulfoxide (DMSO) at a concentration of $10 \mathrm{mM}$ as a stock solution. CAM and AZM were purchased from Tokyo Chemical Industry. Non-antibiotic macrolide EM900 [(8R,9S)8,9- dihydro-6,9- epoxy-8,9- anhydropseudo- erythromycin A] was synthesized as previously reported (25). CAM, AZM and EM900 were dissolved in ethanol to prepare stock solutions of $5 \mathrm{mM}$. E-64d and pepstatin A, which are inhibitors of lysosomal proteases, and pan-caspase inhibitor Z-VAD-FMK were all purchased from Peptide Institute (Osaka, Japan). Necrostatin-1, a specific inhibitor of RIPK1, was purchased from Enzo Life Sciences (Farmingdale, NY, USA).

Cell lines and culture conditions. For the present study, leukemia cell line K562 and pancreatic cancercell lines BxPC-3, Capan-1 and PANC-1 were obtained from the American Type Culture Collection (ATCC; Manassas, VA, USA). BxPC-3 cells and K562 cells were maintained in RPMI-1640 medium (Sigma-Aldrich, St. Louis, MO, USA) supplemented with $10 \%$ fetal bovine serum (FBS; Biowest, Kiltimagh, Ireland), $2 \mathrm{mM}$ L-glutamine, penicillin (100 U/ml), and streptomycin $(100 \mu \mathrm{g} /$ ml) (Wako Pure Chemicals Industries, Osaka, Japan). PANC-1 cells were maintained in Dulbecco's modified Eagle's medium (Sigma) supplemented with $10 \%$ FBS, penicillin $(100 \mathrm{U} / \mathrm{ml})$ and streptomycin $(100 \mu \mathrm{g} / \mathrm{ml})$. Capan-1 cells were maintained in Iscove's modified Dulbecco's medium (Life Technologies, Carlsbad, CA, USA) supplemented with $20 \%$ FBS, penicillin $(100 \mathrm{U} / \mathrm{ml})$ and streptomycin $(100 \mu \mathrm{g} / \mathrm{ml})$. All cell lines were cultured in a humidified incubator containing $5 \% \mathrm{CO}_{2}$ and $95 \%$ air at $37^{\circ} \mathrm{C}$.

Assessment of the viable number of cells. The number of viable cells was assessed using CellTiter Blue, a cell viability assay kit (Promega, Madison, WI, USA), with fluorescence measurements at $570 \mathrm{~nm}$ for excitation and $590 \mathrm{~nm}$ for fluorescence emission.

Immunoblotting. Immunoblotting was performed as previously described (19). Cells were lysed with RIPA lysis 
buffer (Nacalai Tesque, Kyoto, Japan) supplemented with a protease and phosphatase inhibitor cocktail (Nacalai Tesque). Cellular proteins were quantified using a DC protein assay kit of Bio-Rad Laboratories (Richmond, CA, USA). Equal amounts of proteins were loaded onto the gels, separated by SDS-PAGE, and transferred onto Immobilon-P membranes (Millipore Corp., Bedford, MA, USA). These membranes were probed with first antibodies (Abs) such as anti-LC3B antibody (Ab) (Novus Biologicals LLC, Littleton, CO, USA), anti-p62 (D-3) monoclonal (m) Ab (sequestsome-1), anti- $\beta$ actin (C4) $\mathrm{mAb}$, anti-GAPDH (6C5) $\mathrm{mAb}$ (Santa Cruz Biotechnology, Santa Cruz, CA, USA), anticleaved-caspase-3 Ab, and anti-PARP Ab (Cell Signaling Technology, Danvers, MA, USA). Immunoreactive proteins were detected using horseradish peroxidase-conjugated second Abs and an enhanced chemiluminescence reagent (ECL) (Millipore). Densitometry was performed using a Molecular Imager ChemiDoc XRS System (Bio-Rad Laboratories).

Gene expression analysis. Real-time polymerase chain reaction (PCR) for gene expression analysis was performed as previously described in detail (19).

Electron microscopy. Cells were fixed with $2.5 \%$ glutaraldehyde in $0.1 \mathrm{M}$ phosphate buffer ( $\mathrm{pH} 7.3$ ) for $1 \mathrm{~h}$. The samples were further fixed in $1 \%$ osmium tetroxide for $1 \mathrm{~h}$, dehydrated in graded ethanol (30-100\%), and embedded in Quetol 812 epoxy resin (Nisshin EM Co., Ltd., Tokyo, Japan). Ultrathin sections were cut using an Ultracut $\mathrm{J}$ microtome (Reichert Jung, Vienna, Austria). The sections were stained with lead nitrate and uranium acetate, and subjected to electron microscopic analysis using a scanning electron microscope JEM-1200EX II (JEOL, Tokyo, Japan).

Stable transfection of GFP-LC3 plasmid in K562 cells. For the present study, a pEGFP-LC3 plasmid vector (no. 2490) was purchased from Addgene (Cambridge, MA, USA). K562 cells were transfected with plasmid DNA using Lipofectamine 2000 (Life Technologies) according to the manufacturer's instructions. Briefly, $4 \mu \mathrm{g}$ of pEGFP-LC3 plasmids solution and $10 \mu \mathrm{l}$ of Lipofectamine 2000 were incubated in $500 \mu \mathrm{l}$ of serum-free Opti-MEM (Life Technologies) for $20 \mathrm{~min}$ and mixed into $2 \times 10^{6}$ cells cultured in $1.5 \mathrm{ml}$ of antibiotic-free RPMI-1640 with $10 \%$ FBS in a $60-\mathrm{mm}$ dish. Forty-eight hours after transfection, the cells were seeded into a 96 -well plate and selected for limited dilution cloning in the presence of $1 \mu \mathrm{g} / \mathrm{ml}$ of G418 for 1 month. K562/GFP-LC3 clone \#2 was used for the following experiments.

After treatment with macrolide, K562/GFP-LC3 cells were spread and fixed on slide glasses using a Cytospin 4 Centrifuge (Thermo Fisher Scientific, Inc., Rockford, IL, USA) to make slide glass preparations. Analysis by confocal microscopy for the assessment of autophagosomes was performed using a confocal laser scanning fluorescence microscope LSM 700 (Carl Zeiss, Jena, Germany).

Statistical analysis. The data are expressed as mean \pm SD. Statistical analysis was performed using the Mann-Whitney U test (two-tailed).
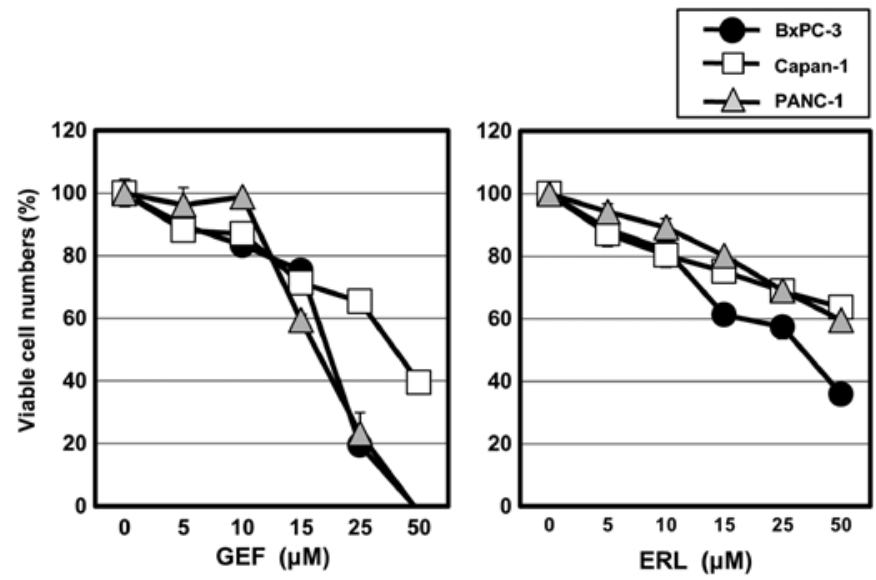

Figure 1. Cell growth inhibition after treatment with GEF and ERL in pancreatic cancer cell lines. BxPC-3, Capan-1 and PANC-1 cells were treated with GEF and ERL at various concentrations for $48 \mathrm{~h}$. The number of viable cells was assessed using CellTiter Blue as described in Materials and methods.

\section{Results}

Cell growth inhibition in pancreatic cancer cell lines after treatment with EGFR-TKI. Pancreatic cancer cell lines PANC-1 and Capan-1, both of which have activating EGFR mutations, and BxPC-3 expressing wild-type EGFR, were cultured in the presence of the EGFR-TKIs GEF and erlotinib hydrochloride (ERL) at various concentrations (Fig. 1). Although both GEF and ERL inhibited cell growth in these cell lines in a dose-dependent manner, GEF was more effective than erlotinib in the cytotoxic effect in our culture system. Therefore, the following in vitro experiments were performed using mainly GEF. A concentration of $50 \%$ cell growth inhibition $\left(\mathrm{IC}_{50}\right)$ after 48 -h treatment with $\mathrm{GEF}$ was $20.4 \mu \mathrm{M}$ for PANC-1, 39.5 $\mu \mathrm{M}$ for Capan-1 and 19.5 $\mu \mathrm{M}$ for BxPC-3.

Autophagy induction in response to GEF in pancreatic cancer cell lines. Conversion from the soluble cytosolic LC3B-I to the membrane-bound LC3B-II via conjugation of phosphatidyl ethanolamine represents the formation of autophagosomes. Therefore, increased expression of LC3B-II is a good marker for autophagosome evaluation (27). Treatment with GEF induced the increased expression of LC3B-II in a dose- and a time-dependent manner in PANC-1 cells and BxPC-3 cells (Fig. 2A). Not shown is that ERL-treatment also increased the expression of LC3B-II as well as GEF. In addition, combined treatment with GEF and lysosomal inhibitors such as E-64d and pepstatin A, which are used for blocking autophagy flux, further enhanced LC3B-II expression compared to treatment with GEF alone or with only lysosomal inhibitors (Fig. 2B). Furthermore, electron microscopy indicated a marked increase of autophagosomes and autolysosomes in PANC-1 cells after treatment with GEF (Fig. 2C). These data indicate that GEF induces autophagy in pancreatic cell lines as well as other cancer cell lines, including NSCLC cells previously reported by us and others $(25,26)$.

Enhanced cytotoxicity of EGFR-TKI by combined treatment with macrolides in pancreatic cancer cell lines. Using NSCLC 
A

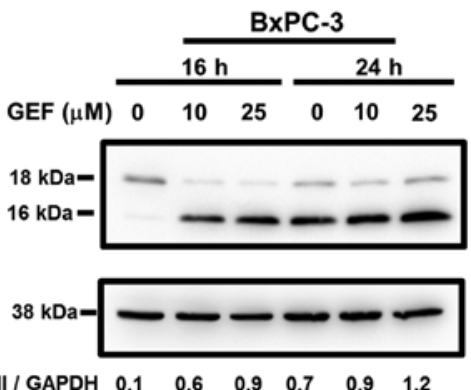

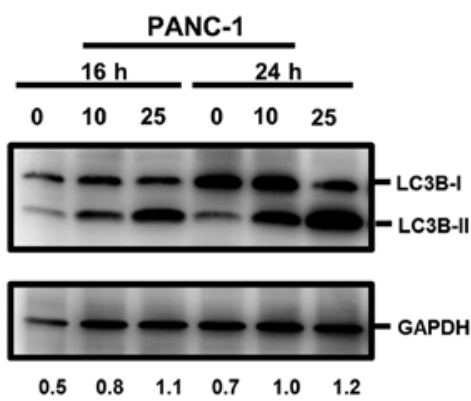

B

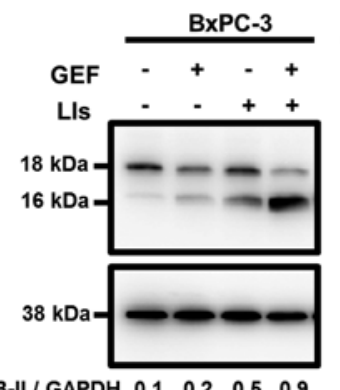

LC3B-II / GAPDH $0.1 \quad 0.2 \quad 0.5 \quad 0.9$
PANC-1

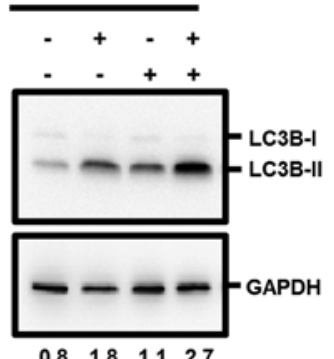

$\begin{array}{llll}0.8 & 1.8 & 1.1 & 2.7\end{array}$
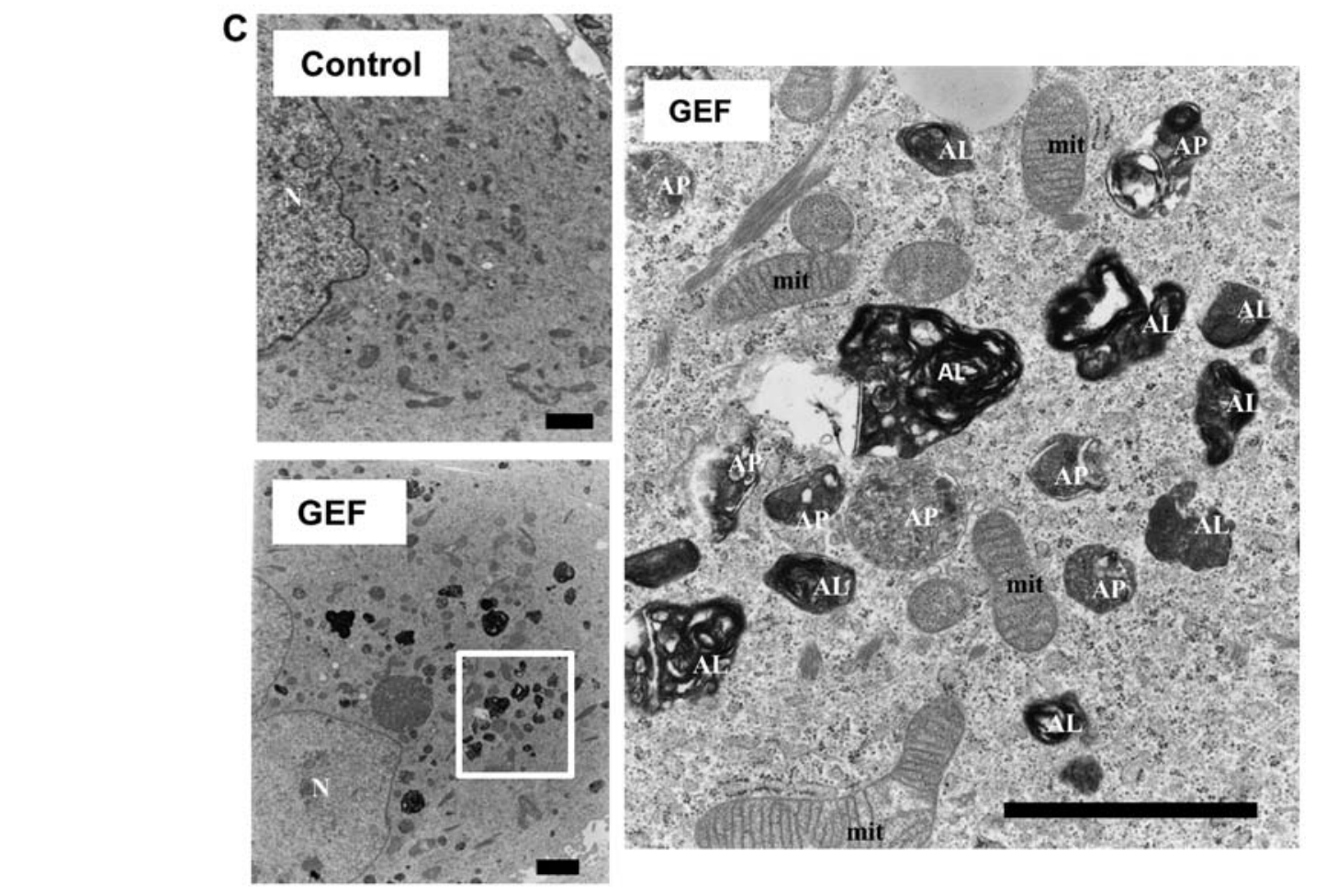

Figure 2. Autophagy induction after treatment with GEF. (A) PANC-1 cells and BxPC-3 cells were treated with GEF (10 and $25 \mu \mathrm{M})$ for 16 and $24 \mathrm{~h}$. Cellular proteins were separated by $15 \%$ SDS-PAGE and immunoblotted with anti-LC3B Ab. Immunoblotting with anti-GAPDH mAb was performed as an internal control. Numbers indicating the expression ratios of LC3B-II/GAPDH were determined using densitometry. (B) PANC-1 cells and BxPC-3 cells were cultured with or without GEF $(25 \mu \mathrm{M})$ in the presence or absence of lysosomal inhibitors (LI), E-64d $(10 \mu \mathrm{g} / \mathrm{ml})$, and pepstatin A $(10 \mu \mathrm{g} / \mathrm{ml})$ for 16 and $24 \mathrm{~h}$. Immunoblottings were performed as (A). Numbers indicate the expression ratios of LC3B-II/GAPDH. (C) PANC-1 cells were treated with GEF (25 $\mu \mathrm{M})$ for $48 \mathrm{~h}$, and electron microscopy was performed. Scale bars, $2 \mu \mathrm{m}$. N, nucleus; mit, mitochondria; AP, autophagosome; AL, autolysosome.

cell lines, we have reported that GEF-induced autophagy functions as cytoprotective, and simultaneous treatment with GEF and CAM results in enhanced cytotoxicity (25). In the present study, we selected three macrolides (CAM, AZM and EM900) and examined their efficacy for GEF-induced cytotoxicity as well as blocking autophagy flux in pancreatic cancer cell lines. First, treatment with up to $50 \mu \mathrm{M}$ CAM, AZM or EM900 resulted in little cytotoxicity in BxPC-3 cells. More than $75 \mu \mathrm{M}$ EM900 exhibited some cytotoxic effect (Fig. 3A). Next, BxPC-3 and PANC-1 cells were treated with GEF at various concentrations in the presence of $50 \mu \mathrm{M} \mathrm{CAM}$, AZM or EM900. Significant enhancement of GEF-induced cytotoxicity was observed (Fig. 3B). During 48-h exposure, AZM was more potent than CAM or EM900 for enhancing GEF-induced cytotoxicity. At 25 and $50 \mu \mathrm{M}$ GEF, EM900 was superior to CAM. The concentration of GEF was therefore fixed at $25 \mu \mathrm{M}$, and PANC-1 cells were treated with macrolides at various concentrations. It was noteworthy that all three macrolides at $>5 \mu \mathrm{M}$ resulted in enhanced reduction of the viable cell number as comparing with treating the cells with GEF alone (Fig. 3C). Additionally, AZM was superior to CAM and EM900 in enhancing the killing effect of $25 \mu \mathrm{M}$ GEF.

Comparing the blocking effect of autophagy flux among CAM, AZM and EM900 in PANC-1 cells. Based on the above data, we further assessed the blocking effect of macrolides on autophagy flux in pancreatic cancer cell lines. After treatment with $50 \mu \mathrm{M}$ macrolides for 24 and $48 \mathrm{~h}, \mathrm{PANC}-1$ cells exhibited increased expression of LC3B-II (Fig. 4A). However, unlike GEF treatment (Fig. 2B), combined treatment with lysosomal inhibitors and a macrolide did not increase LC3B-II expression further, compared with treatment with either a macrolide alone or only lysosome inhibitors. These results indicate that all tested macrolides effectively block autophagy flux (Fig. 4B). For comparison of efficiency on blocking autophagy, PANC-1 cells were treated with a macrolide at various concentrations 
A

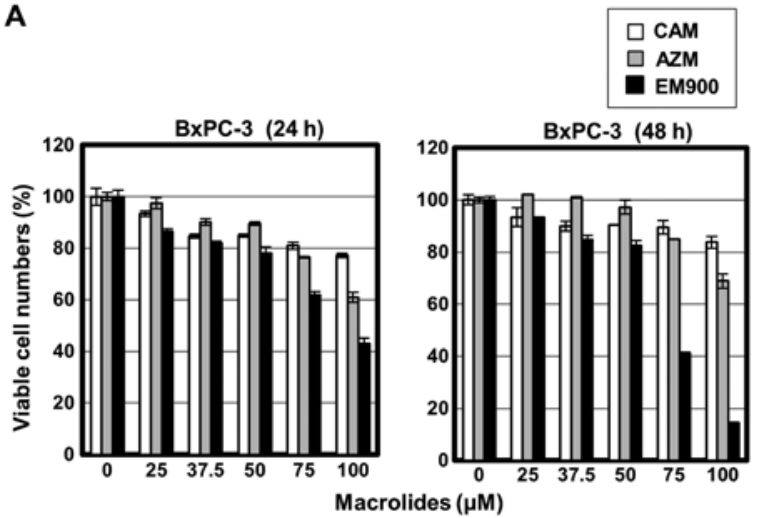

B
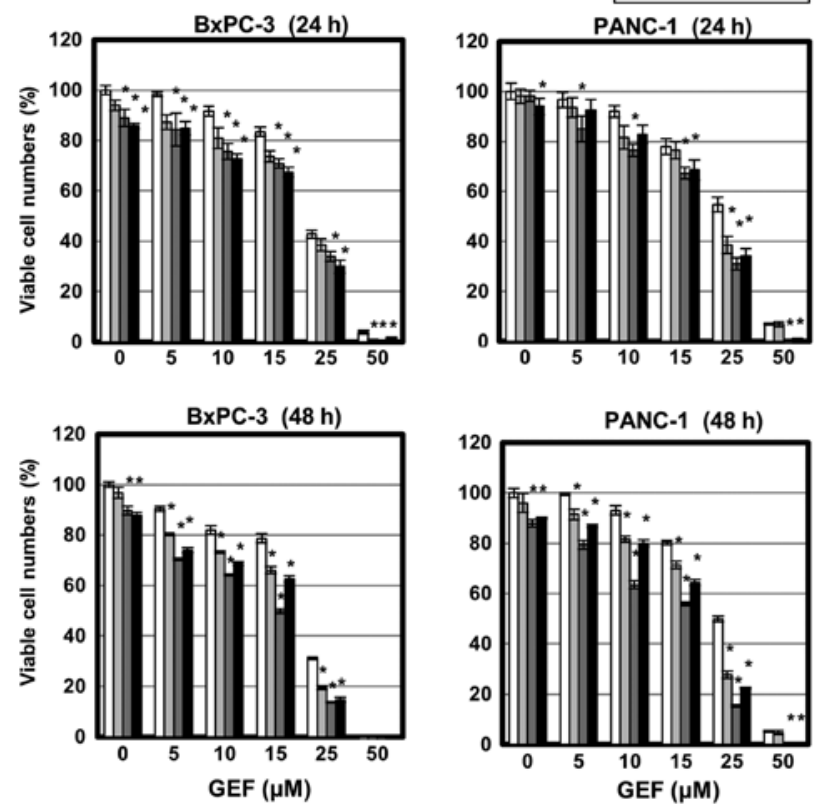

C
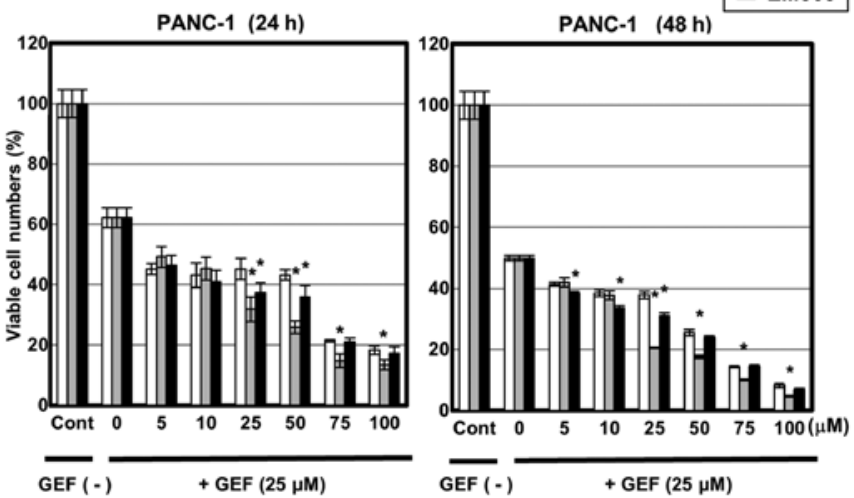

Figure 3. Enhancement of cell growth inhibition after combined treatmen with GEF and macrolides in PANC-1 and BxPC-3 cells. (A) BxPC-3 cells were treated with CAM, AZM and EM900 at various concentrations for 24 and $48 \mathrm{~h}$. The number of viable cells was then assessed using CellTiter Blue. (B) BxPC-3 and PANC-1 cells were treated with GEF at various concentrations in the presence or absence of macrolides $(50 \mu \mathrm{M})$ for 24 and $48 \mathrm{~h}$ ${ }^{*} \mathrm{P}<0.05$ control vs. macrolide. (C) PANC-1 cells were treated with macrolides at various concentrations in the presence or absence of GEF $(25 \mu \mathrm{M})$ for 24 and $48 \mathrm{~h}$. The number of viable cells was assessed using CellTiter Blue. ${ }^{*} \mathrm{P}<0.05 ;$ CAM vs. AZM/EM900.
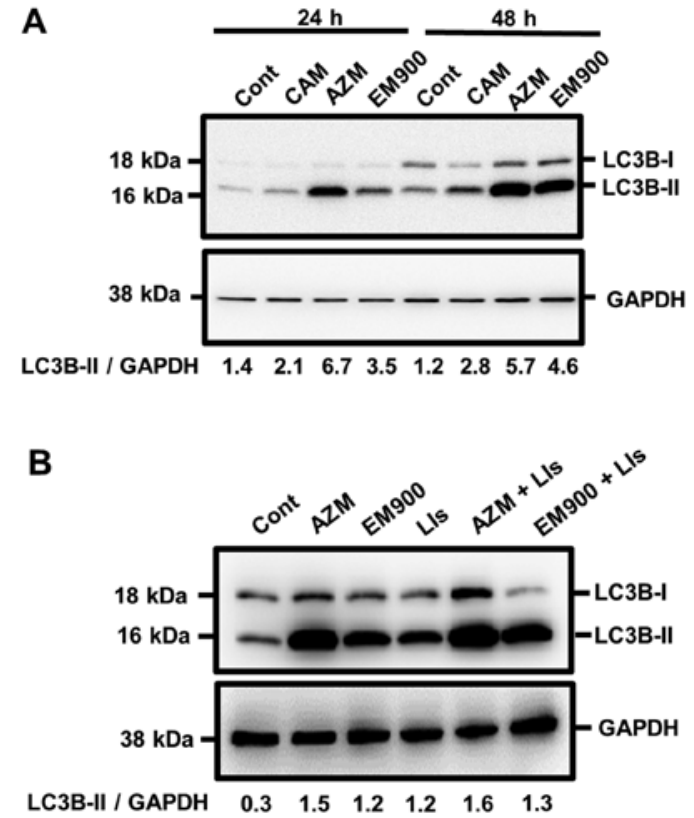

Figure 4. Flux analysis of autophagosome formation in macrolides (A) PANC-1 cells were treated with CAM, AZM and EM900 at $50 \mu \mathrm{M}$ for 24 and $48 \mathrm{~h}$. Cellular proteins were separated by $15 \%$ SDS-PAGE and immunoblotted with anti-LC3B Ab. Immunoblotting with anti-GAPDH mAb was performed as an internal control for protein loading. Numbers indicate the expression ratios of LC3B-II/GAPDH. (B) PANC-1 cells were treated with AZM and EM900 at $50 \mu \mathrm{M}$ in the presence or absence of lysosomal inhibitors (LIs) for $16 \mathrm{~h}$, as depicted in Fig. 2B. Immunoblotting was performed as in (A). Numbers indicate the expression ratios of LC3B-II/GAPDH.

for $24 \mathrm{~h}$. Immunoblotting with anti-LC3B Ab clearly indicated accumulation of autophagosome in a dose-dependent manner for all three macrolides (Fig. 5A). P62, a substrate of autophagy (28), also accumulated after macrolide treatment in a dose-dependent manner. To standardize for comparison among the three macrolides, the expression ratios of LC3B-II to GAPDH were divided by that of untreated controls and plotted (Fig. 5B). AZM is the most potent in blocking efficiency in autophagy; EM900 is the second most potent and superior to CAM. It was noteworthy that the inhibitory efficacy of autophagy in macrolides correlated well with their enhancing effect for GEF-induced cytotoxicity (Fig. 3B and C). To confirm their blocking efficiency, K562 cells with a stably transfected GFP-LC3 gene (K562/GFP-LC3) were cultured in the presence of these macrolides at $50 \mu \mathrm{M}$ for $24 \mathrm{~h}$. As indicated in Fig. 5C and D, the number of puncta of GFP-LC3 pre-cell, which indicates autophagosome accumulation in response to the macrolide, was consistent with the result presented in Fig. 5A and B. Increase in the number of GFP-LC3 puncta was in the order of AZM, EM900 and CAM. These data together strongly suggested that enhanced GEF-induced cytotoxicity by combining macrolides is due to their effect in blocking autophagy flux.

Non-apoptotic cell death induction after treatment with GEF with/without macrolides. We next assessed ER stress loading after treatment with GEF and macrolides, because apoptosis is induced when ER stress is overloaded beyond cellular adaptive capacity $(21,22)$. Real-time PCR indicated that GEF treatment upregulated ER stress-related genes such as GRP78 and 
A
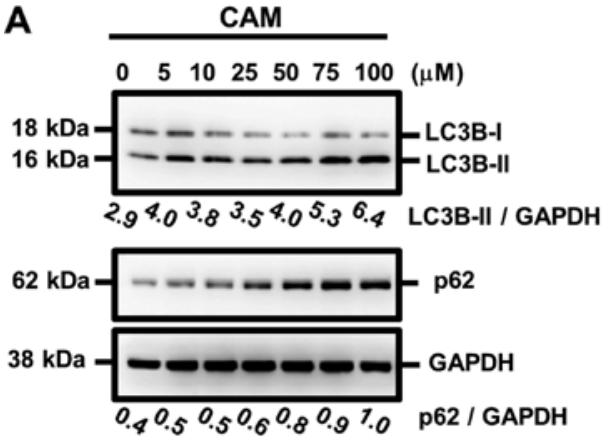

AZM
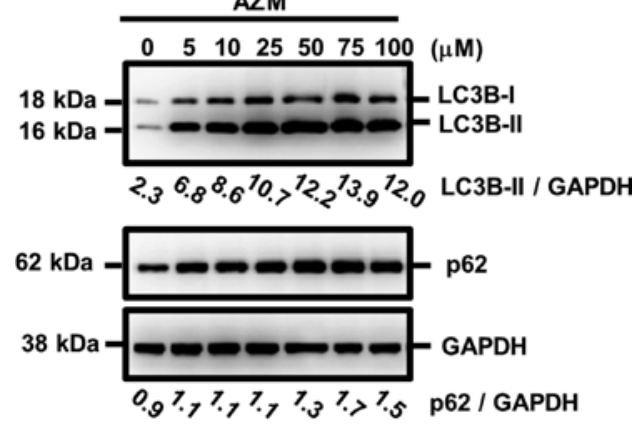

EM900

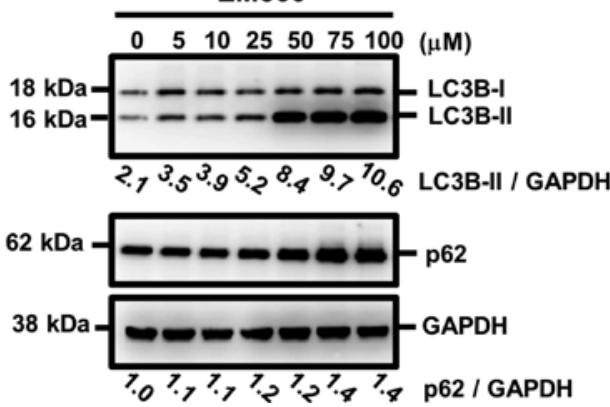

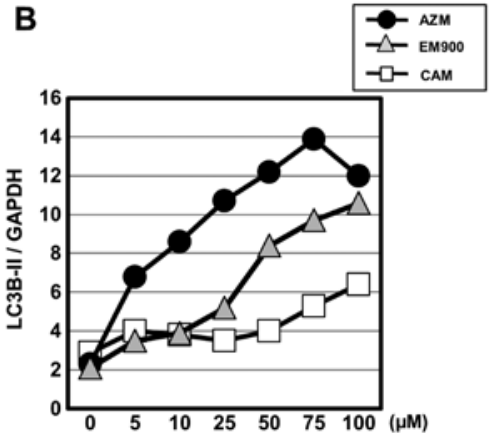

C Control
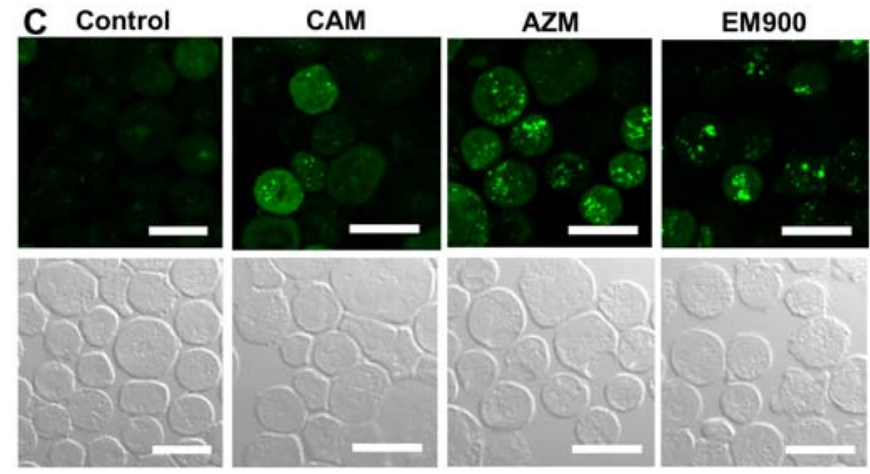

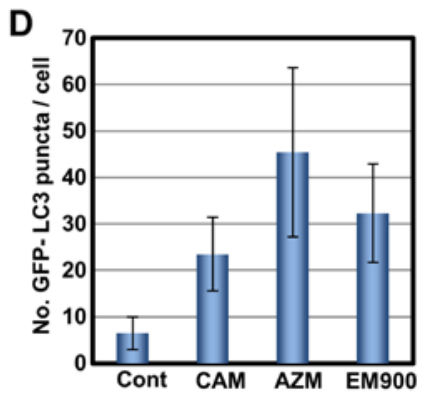

Figure 5. Comparison of macrolides in the efficiency of blocking autophagy flux. (A) PANC-1 cells were treated with AZM, EM900 and CAM at various concentrations for $24 \mathrm{~h}$. Cellular proteins were separated by 15\% SDS-PAGE for LC3B and $11.25 \%$ for p62 and immunoblotted with anti-LC3B and anti-p62 mAbs. Immunoblotting with anti-GAPDH mAb was performed as an internal control. Numbers indicate the ratios of LC3B-II/GAPDH and p62/GAPDH as determined using densitometry. (B) Expression ratios of LC3B-II/GAPDH presented above were plotted. (C) Confocal microscopy: K562-GFP-LC3 cells were treated with $50 \mu \mathrm{M}$ AZM, EM900 and CAM for $24 \mathrm{~h}$. Upper panels, puncta of green fluorescence indicates accumulating autophagosome. Lower panels, differential interference contrast microscopy. Scale bars, $10 \mu \mathrm{m}$. (D) Numbers of puncta of GFP-LC3 per cell in Fig. 5C. CAM vs. AZM, P<0.001; AZM vs. EM900, $\mathrm{P}=0.02$; EM900 vs. CAM, $\mathrm{P}=0.03$.
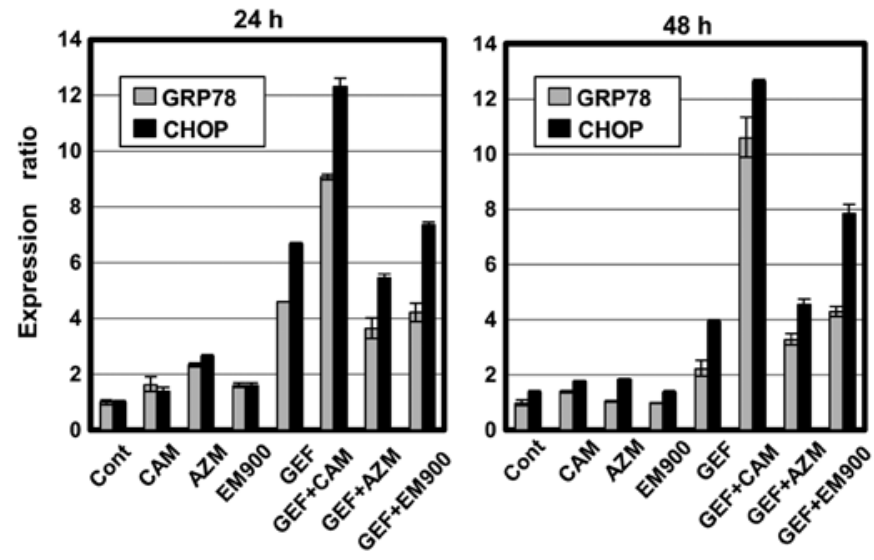

Figure 6. Induction of GRP78 and CHOP in PANC-1 cells after treatment with GEF and macrolides. PANC-1 cells were treated with/without GEF in the presence or absence of the three macrolides for 24 and $48 \mathrm{~h}$. Expressions of ER stress-related genes GRP78 and CHOP were assessed using real-time PCR.
CHOP, whereas, treatment with macrolides had little effect (Fig. 6). Combined treatment with GEF plus CAM resulted in pronounced expressions of GRP78 and CHOP, which is a pro-apoptotic transcription factor that upregulates various pro-apoptotic genes such as Bax and Bim (21). Unexpectedly, concomitant AZM or EM900 plus GEF did not significantly enhance ER stress loading compared with treatment of the cells with GEF alone. A discrepancy between the efficiency of autophagy inhibition and ER stress loading led us to examine whether the enhanced cytotoxicity is completely mediated though apoptosis.

As indicated in Fig. 7A, treating PANC-1 cells with GEF in the presence or absence of EM900 produced no apoptotic features but did produce necrosis. However, cells treated with gemcitabine (gem), which is the most widely used medicine for treating patients with metastatic pancreatic cancer, produced nuclear chromatin condensation in some cells (arrows). 

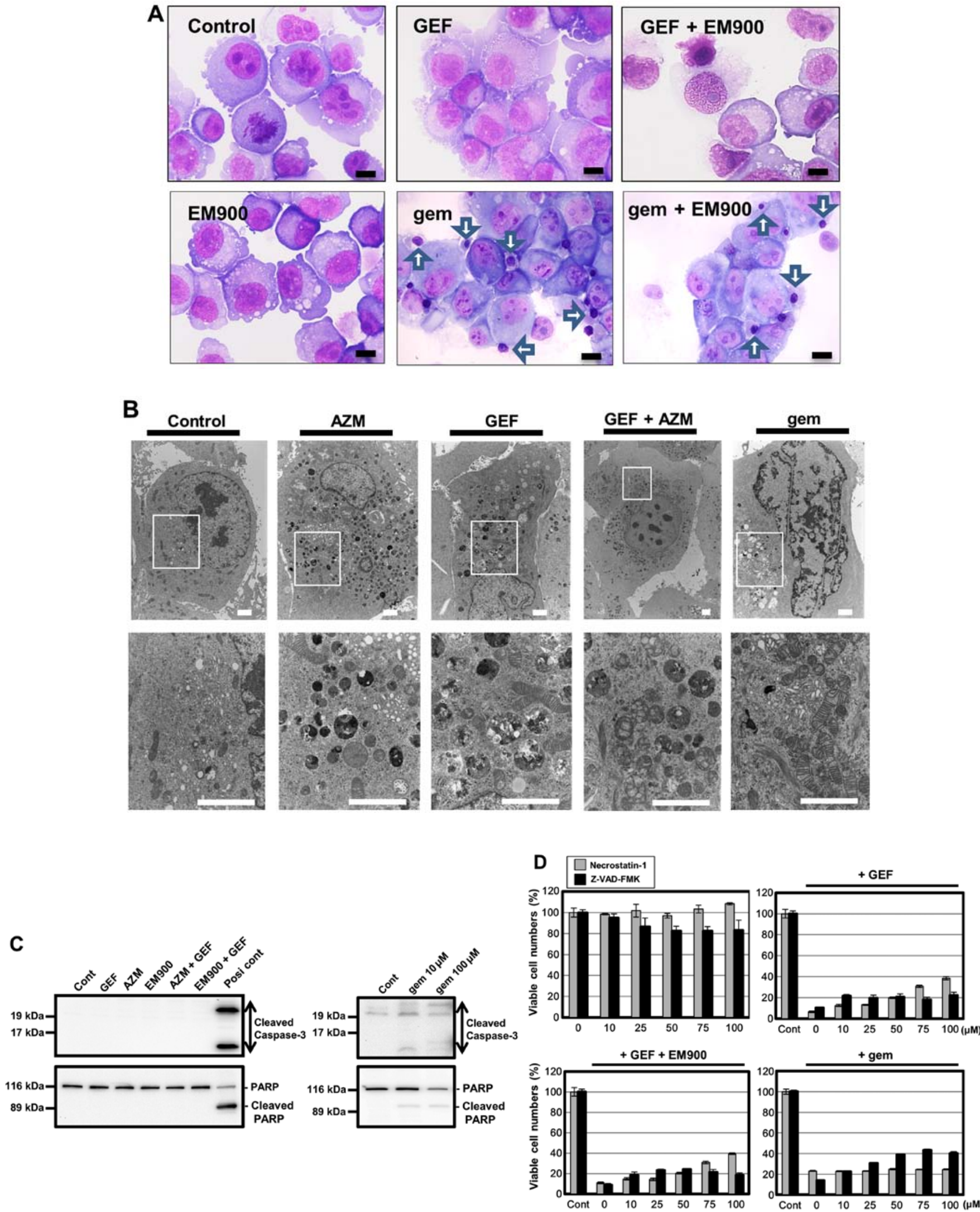

Figure 7. Morphological changes in PANC-1 cells after treatment with GEF and macrolides. (A) May-Giemsa staining: May-Giemsa staining was performed after treatment with either GEF $(25 \mu \mathrm{M})$ or gemcitabine $(100 \mu \mathrm{M})$ in the presence or absence of EM900 $(50 \mu \mathrm{M})$ for $48 \mathrm{~h}$. Scale bars, $10 \mu \mathrm{m}$. Arrows indicate nuclear chromatin condensations. (B) Electron microscopy: PANC-1 cells were processed for electron microscopy after treatment with GEF ( $25 \mu \mathrm{M}), \mathrm{AZM}$ $(50 \mu \mathrm{M}), \mathrm{GEF}+\mathrm{AZM}$, and gemcitabine $(100 \mu \mathrm{M})$ for $48 \mathrm{~h}$. Lower panels are at higher magnifications of the squared areas in each upper panel. Scale bars, $2 \mu \mathrm{m}$. (C) PANC-1 cells were treated with GEF $(25 \mu \mathrm{M})$, AZM $(50 \mu \mathrm{M})$, EM900 $(50 \mu \mathrm{M})$, GEF+AZM, and gemcitabine (10 and $100 \mu \mathrm{M})$ for $48 \mathrm{~h}$. Cellular proteins were separated by $15 \%$ SDS-PAGE for caspase-3 and 7.5\% for PARP and immunoblotted with either anti-cleaved caspase-3 Ab or anti-PARP Ab. Cell lysate derived from HL-60 cells treated with vitamin K2 was used as a positive control for apoptosis induction (42). (D) PANC-1 cells were treated with various concentrations of either necrostatin-1 or Z-VAD-FMK in the presence or absence of GEF (50 $\mu \mathrm{M})$, GEF (50 $\mu \mathrm{M})$ plus EM900 (50 $\mu \mathrm{M})$, or gem (300 $\mu \mathrm{M})$ for $48 \mathrm{~h}$. The number of viable cells was assessed using CellTiter Blue. 
Electron microscopy also indicated that, although gem-treated PANC-1 cells exhibited a sharp nuclear notch as well as nuclear heterochromatin formation GEF treatment with/without AZM did not produce prominent nuclear change, but did produce accumulation of a large number of autophagosomes and autolysosomes, and increased swollen mitochondria with destruction of cristae structure inside them (Fig. 7B). Furthermore, immunoblotting with anti-cleaved capase-3 Ab and anti-PARP Ab indicated no cleavage of caspase-3 and PARP after treatment with GEF plus AZM or EM900 (Fig. 7C). All these data suggest that enhanced cytotoxicity by combining GEF plus macrolide as well as GEF-induced cytotoxicity itself does not involve apoptosis in pancreatic cancer cell lines. This result was also supported by the data presented in Fig. 7D; in the presence of necrostatin-1, a potent and selective inhibitor of necroptosis via blocking RIP-1 kinase (29), GEF- and GEF plus EM900-induced cell death was significantly attenuated, while Z-VAD-FMK, a pan-caspase inhibitor for apoptosis inhibition, had little effect. In contrast, gemcitabine-induced cytotoxicity was significantly suppressed in the presence of Z-VAD-FMK, but not in the presence of necrostatin-1. Thus, a combination of GEF plus macrolide induces mainly non-apoptosis cell death such as necroptosis, whereas GEM induces apoptosis, in PANC-1 cells.

\section{Discussion}

In the present study, we demonstrated that macrolides such as AZM, CAM and EM900 enhance GEF-induced cytotoxicity in pancreatic cancer cell lines. In addition, the efficiency of macrolides in blocking autophagy flux correlated well with their enhancement of GEF-induced cytotoxicity (Figs. 3 and 4). Among the three macrolides, AZM was the most potent for enhancing the GEF cytotoxicity as well as for inhibiting autophagy. Many previous reports suggest that autophagy induction by GEF is a metabolic stress response and functions as cytoprotective, which might be crucial in resistance to EGFR-TKIs (11-13). Therefore, it is reasonable that blocking efficiency directly influences the cytotoxic effect of GEF. However, the precise mechanism for blocking autophagy by macrolides must still be clarified.

Based on the chemical structure of macrolides, a common target appears to be involved in the process of autophagy. Autophagy inhibition by AZM was originally reported by Renna et al in 2011 (18). Long-term oral administration of AZM against chronic sinopulmonary infection in adult patients with cystic fibrosis is associated with the development of infection with non-tuberculous mycobacteria. They found that, in primary human macrophages, AZM concentrations achieved during therapeutic dosing blocked autophagosome clearance by preventing lysosomal acidification, thereby impairing autophagic and phagosomal degradation. As a consequence, AZM treatment inhibited intracellular killing of mycobacteria within macrophages, which resulted in chronic non-tuberculous mycobacteria infection (18). Electron microscopy indicated accumulation of a large number of autophagosomes and autolysosomes in PANC-1 cells after treatment with AZM (Fig. 7B). This result indicated that the membrane fusion between autophagosome and lysosome (autolysosome formation) is not impaired.
Lysosomes generate and maintain their $\mathrm{pH}$ gradients by vacuolar (V)-type ATPase activity for pumping protons into the lysosome lumen (31). Therefore, preventing lysosomal acidification by AZM as indicated by a previous report suggests that V-type ATPase is a candidate target molecule for AZM. Indeed, bafilomycin $\mathrm{A}_{1}$ and concanamycin $\mathrm{A}$ are macrolide compounds that have a selective inhibitory effect on V-type ATPase, and they are frequently used in experiments in vitro for blocking autophagy (32). However, both macrolides have reportedly prevented maturation of autophagic vacuoles by inhibiting fusion between autophagosomes and lysosomes (32). Valosin-containing protein (VCP)/p97 appears to be another potential target (33). A recent report demonstrated that AZM and CAM interact with VCP, based on AZM- and CAM-immobilized affinity chromatography (33). Pathophysiologically, germline mutations in VCP genes account for a spectrum of pathological phenotypes that include inclusion body myopathy with Paget's disease of the bone and frontotemporal dementia, hereditary spastic paraplegia (IBMPFD), and 1-2\% of familial amyotrophic lateral sclerosis $(34,35)$. In addition, $\mathrm{VCP} / \mathrm{p} 97$ plays a critical role in a broad range of cellular activities, including ERAD, the ubiquitin-proteasome system, prevention of polyglutamine aggregation and autophagosome maturation in autophagy $(35,36)$. VCP mutation (p.Glu185Lys) segregating in the autosomal dominant Charcot-Marie-Tooth disease type 2 family was also identified (37). Notably, functional studies confirmed that the Glu185Lys variant impaired autophagic function, leading to accumulation of immature autophagosomes (37). Identification of the target molecule(s) of macrolide for autophagy inhibition as well as assessment of the affinity to the macrolides used in this study helps to clarify the phenomena analyzed in this study.

Despite the positive relationship between blocking efficiency and enhancement of the cytotoxicity of GEF, discrepancies in ER stress loading were observed (Fig. 5 vs. 6). CAM and GEF was the most potent combination for upregulation of pro-apoptotic transcription factor $\mathrm{CHOP}$, whereas concomitant AZM or EM900 plus GEF did not significantly enhance ER stress loading, compared with GEF alone (Fig. 6). Other than ER stress-mediated apoptosis, alternative pathway(s) such as non-apoptotic cell death might contribute to the pronounced cytotoxic effect. We previously reported that a murine embryonic fibroblast (MEF) cell line derived from CHOP knockout mice still exhibited enhanced cytotoxicity with combined treatment of GEF and CAM, although they were much less sensitive to GEF plus CAM than the $\mathrm{CHOP}^{+/+} \mathrm{MEF}$ cell line (24). The same phenomenon was observed in PC-9 cell knocked down CHOP by siRNA versus control PC-9 cells (24). Although cytotoxicity was enhanced, a series of experiments performed for assessing apoptosis did not yield any apparent features of apoptosis (Fig. 7A-C). However, in the presence of necrostatin-1, a specific inhibitor for necroptosis via blocking RIP1 kinase (29), GEF- and GEF plus macrolide-induced cell death was partially but significantly suppressed (Fig. 7D). This result suggests that necroptosis might contribute to enhanced cytotoxicity. Unlike NSCLC cell lines, PANC-1 and BxPC-3 cells did not exhibit prominent apoptotic features even after treatment with GEF alone (24). In addition, treatment with gemcitabine resulted in only slight apoptotic change, such as 
weak cleavages of caspase-3 and PARP, as well as partial chromatin condensation without nuclear fragmentation (Fig. 7A-C). Also, their features were much weaker than those in other cell lines (e.g., NSCLC, breast cancer and leukemia cell lines) (data not shown). Therefore, pancreatic cancer cell lines used in the present study may be rather resistant to apoptosis induction. This result might represent the difficult clinical features of pancreatic cancer patients with chemoresistance.

It is necessary to determine the most effective way to interconnect autophagy inhibition and non-apoptotic cell death. Currently, no study has demonstrated necroptosis induction by EGFR-TKIs. However, it has been confirmed that non-apoptotic cell death, including necroptosis, alternatively becomes prominent when cells are resistant to apoptosis induction (38-40). In PANC-1 cells, a higher concentration of gemcitabine $(100 \mu \mathrm{M})$ was required for apoptosis induction, indicating difficulty in inducing apoptosis in them, unlike other cancer cell lines (Fig. 7D). It was reported that autophagy suppresses RIP kinase-dependent necrosis (necroptosis) in RCC4 cells, a human renal cell carcinoma cell line (41). Inhibition of mTOR with the specific mTOR inhibitor CCL-779 stimulated autophagy, leading to elimination of RIP kinases via autophagy-mediated degradation. However, with the simultaneous inhibition of autophagy using chloroquine and mTOR with CCL-779, necroptosis was induced (41). This result is somewhat similar to our observation, since EGFR-TKI inhibits the axis of the PI3K/AKT/mTOR pathway. In their system, autophagy of mitochondria (mitophagy) is required for cell survival, since mTOR inhibition turns off the Nrf2 antioxidant defense (41). Thus, simultaneous mTOR and autophagy inhibition leads to an imbalance between ROS production by mitochondria and the defense by Nrf2, which causes necroptosis. Pancreatic cell lines appear to have higher basal autophagy with increased mitochondria than other cancer cell lines (Figs. 2A and 7B). For clinical use of a macrolide as a potent 'sensitizer' in EGFR-TKI therapy, it will also be important to establish the underlying molecular mechanism of enhanced non-apoptotic cell death, as well as to identify the target(s) of macrolides.

\section{Acknowledgements}

The present study was supported by funds provided through an MEXT-supported program of the Strategic Research Foundation at Private Universities (S1411011, 2014-2018) from the Ministry of Education, Culture, Sports, Science and Technology of Japan to M.H. and K.M., Grants-in-Aid for Scientific Research (C) from The Ministry of Education, Culture, Sports, Science and Technology to T.I. (no. 24591018) and to K.M. (no. 26460478), and a Grant-in-Aid from Tokyo Medical University Cancer Research to K.M.

\section{References}

1. Gresham GK, Wells GA, Gill S, Cameron C and Jonker DJ: Chemotherapy regimens for advanced pancreatic cancer: A systematic review and network meta-analysis. BMC Cancer 14: $471,2014$.

2. Von Hoff DD, Ervin T, Arena FP, Chiorean EG, Infante J, Moore M, Seay T, Tjulandin SA, Ma WW, Saleh MN, et al: Increased survival in pancreatic cancer with nab-paclitaxel plus gemcitabine. N Engl J Med 369: 1691-1703, 2013.
3. Ueda S, Ogata S, Tsuda H, Kawarabayashi N, Kimura M, Sugiura Y, Tamai S, Matsubara O, Hatsuse K and Mochizuki H: The correlation between cytoplasmic overexpression of epidermal growth factor receptor and tumor aggressiveness: Poor prognosis in patients with pancreatic ductal adenocarcinoma. Pancreas 29: e1-e8, 2004.

4. Moore MJ, Goldstein D, Hamm J, Figer A, Hecht JR, Gallinger S, Au HJ, Murawa P, Walde D, Wolff RA, et al; National Cancer Institute of Canada Clinical Trials Group: Erlotinib plus gemcitabine compared with gemcitabine alone in patients with advanced pancreatic cancer: A phase III trial of the National Cancer Institute of Canada Clinical Trials Group. J Clin Oncol 25: 1960-1966, 2007.

5. Yang ZY, Yuan JQ, Di MY, Zheng DY, Chen JZ, Ding H, Wu XY, Huang YF, Mao C and Tang JL: Gemcitabine plus erlotinib for advanced pancreatic cancer: A systematic review with metaanalysis. PLoS One 8: e57528, 2013.

6. Diaz Beveridge R, Alcolea V, Aparicio J, Segura Á, García J, Corbellas M, Fonfría M, Giménez A and Montalar J: Management of advanced pancreatic cancer with gemcitabine plus erlotinib: Efficacy and safety results in clinical practice. JOP 15: 19-24, 2014.

7. Mizushima N, Levine B, Cuervo AM and Klionsky DJ: Autophagy fights disease through cellular self-digestion. Nature 451: 1069-1075, 2008.

8. Galluzzi L, Pietrocola F, Levine B and Kroemer G: Metabolic control of autophagy. Cell 159: 1263-1276, 2014.

9. White E: Deconvoluting the context-dependent role for autophagy in cancer. Nat Rev Cancer 12: 401-410, 2012.

10. Ciardiello $F$ and Tortora G: EGFR antagonists in cancer treatment. N Engl J Med 358: 1160-1174, 2008.

11. Han W, Pan H, Chen Y, Sun J, Wang Y, Li J, Ge W, Feng L, Lin X, Wang X, et al: EGFR tyrosine kinase inhibitors activate autophagy as a cytoprotective response in human lung cancer cells. PLoS One 6: e18691, 2011.

12. Jutten B and Rouschop KM: EGFR signaling and autophagy dependence for growth, survival, and therapy resistance. Cell Cycle 13: 42-51, 2014.

13. Fung C, Chen X, Grandis JR and Duvvuri U: EGFR tyrosine kinase inhibition induces autophagy in cancer cells. Cancer Biol Ther 13: 1417-1424, 2012.

14. Kim YC and Guan KL: mTOR: A pharmacologic target for autophagy regulation. J Clin Invest 125: 25-32, 2015.

15. Wei Y, Zou Z, Becker N, Anderson M, Sumpter R, Xiao G, Kinch L, Koduru P, Christudass CS, Veltri RW, et al: EGFRmediated Beclin 1 phosphorylation in autophagy suppression, tumor progression, and tumor chemoresistance. Cell 154: $1269-1284,2013$.

16. Tan X, Thapa N, Sun Y and Anderson RA: A kinase-independent role for EGF receptor in autophagy initiation. Cell 160: 145-160, 2015.

17. Nakamura M, Kikukawa Y, Takeya M, Mitsuya $\mathrm{H}$ and Hata $\mathrm{H}$ : Clarithromycin attenuates autophagy in myeloma cells. Int $\mathrm{J}$ Oncol 37: 815-820, 2010.

18. Renna M, Schaffner C, Brown K, Shang S, Tamayo MH, Hegyi K, Grimsey NJ, Cusens D, Coulter S, Cooper J, et al: Azithromycin blocks autophagy and may predispose cystic fibrosis patients to mycobacterial infection. J Clin Invest 121: 3554-3563, 2011.

19. Moriya S, Che XF, Komatsu S, Abe A, Kawaguchi T, Gotoh A, Inazu M, Tomoda A and Miyazawa K: Macrolide antibiotics block autophagy flux and sensitize to bortezomib via endoplasmic reticulum stress-mediated CHOP induction in myeloma cells. Int J Oncol 42: 1541-1550, 2013.

20. Moriya S, Komatsu S, Yamasaki K, Kawai Y, Kokuba H, Hirota A, Che XF, Inazu M, Gotoh A, Hiramoto M, et al: Targeting the integrated networks of aggresome formation, proteasome, and autophagy potentiates ER stress-mediated cell death in multiple myeloma cells. Int J Oncol 46: 474-486, 2015.

21. Verfaillie T, Salazar M, Velasco G and Agostinis P: Linking ER stress to autophagy: Potential implications for cancer therapy. Int J Cell Biol 2010: 930509, 2010.

22. Wang $M$ and Kaufman RJ: The impact of the endoplasmic reticulum protein-folding environment on cancer development. Nat Rev Cancer 14: 581-597, 2014.

23. Tabas I and Ron D: Integrating the mechanisms of apoptosis induced by endoplasmic reticulum stress. Nat Cell Biol 13: 184-190, 2011.

24. Sugita S, Ito K, Yamashiro Y, Moriya S, Che XF, Yokoyama T, Hiramoto $M$ and Miyazawa K: EGFR-independent autophagy induction with gefitinib and enhancement of its cytotoxic effect by targeting autophagy with clarithromycin in non-small cell lung cancer cells. Biochem Biophys Res Commun 461: 28-34, 2015. 
25. Sugawara A, Sueki A, Hirose T, Nagai K, Gouda H, Hirono S Shima H, Akagawa KS, Omura S and Sunazuka T: Novel 12-membered non-antibiotic macrolides from erythromycin A; EM900 series as novel leads for anti-inflammatory and/or immunomodulatory agents. Bioorg Med Chem Lett 21: 3373-3376, 2011.

26. Dragowska WH, Weppler SA, Wang JC, Wong LY, Kapanen AI, Rawji JS, Warburton C, Qadir MA, Donohue E, Roberge M, et al: Induction of autophagy is an early response to gefitinib and a potential therapeutic target in breast cancer. PLoS One 8: e76503, 2013

27. Mizushima N and Yoshimori T: How to interpret LC3 immunoblotting. Autophagy 3: 542-545, 2007.

28. Jiang P and Mizushima N: LC3- and p62-based biochemical methods for the analysis of autophagy progression in mammalian cells. Methods 75: 13-18, 2015.

29. Degterev A, Hitomi J, Germscheid M, Ch'en IL, Korkina O, Teng X, Abbott D, Cuny GD, Yuan C, Wagner G, et al: Identification of RIP1 kinase as a specific cellular target of necrostatins. Nat Chem Biol 4: 313-321, 2008.

30. Sui X, Kong N, Zhu M, Wang X, Lou F, Han W and Pan H: Cotargeting EGFR and autophagy signaling: A novel therapeutic strategy for non-small-cell lung cancer. Mol Clin Oncol 2: 8-12, 2014.

31. Mindell JA: Lysosomal acidification mechanisms. Annu Rev Physiol 74: 69-86, 2012.

32. Harada M, Sakisaka S, Yoshitake M, Kin M, Ohishi M, Shakado S, Mimura Y, Noguchi K, Sata M and Tanikawa K: Bafilomycin A1, a specific inhibitor of vacuolar-type $\mathrm{H}(+)$-ATPases, inhibits the receptor-mediated endocytosis of asialoglycoproteins in isolated rat hepatocytes. J Hepatol 24: 594-603, 1996.

33. Nujić K, Smith M, Lee M, Belamarić D, Tomašković L, Alihodžić S, Malnar I, Polančec D, Schneider K and Eraković Haber V: Valosin containing protein (VCP) interacts with macrolide antibiotics without mediating their anti-inflammatory activities. Eur J Pharmacol 677: 163-172, 2012.
34. Watts GD, Wymer J, Kovach MJ, Mehta SG, Mumm S, Darvish D, Pestronk A, Whyte MP and Kimonis VE: Inclusion body myopathy associated with Paget disease of bone and frontotemporal dementia is caused by mutant valosin-containing protein. Nat Genet 36: 377-381, 2004.

35. Meyer $\mathrm{H}$ and Weihl CC: The VCP/p97 system at a glance: Connecting cellular function to disease pathogenesis. J Cell Sci 127: 3877-3883, 2014.

36. Meyer H, Bug M and Bremer S: Emerging functions of the VCP/ p97 AAA-ATPase in the ubiquitin system. Nat Cell Biol 14: 117-123, 2012.

37. Gonzalez MA, Feely SM, Speziani F, Strickland AV, Danzi M, Bacon C, Lee Y, Chou TF, Blanton SH, Weihl CC, et al: A novel mutation in VCP causes Charcot-Marie-Tooth Type 2 disease. Brain 137: 2897-2902, 2014.

38. Nikoletopoulou V, Markaki M, Palikaras K and Tavernarakis N: Crosstalk between apoptosis, necrosis and autophagy. Biochim Biophys Acta 1833: 3448-3459, 2013.

39. Vanden Berghe T, Linkermann A, Jouan-Lanhouet S, Walczak H and Vandenabeele P: Regulated necrosis: The expanding network of non-apoptotic cell death pathways. Nat Rev Mol Cell Biol 15: 135-147, 2014.

40. Feoktistova $M$ and Leverkus $M$ : Programmed necrosis and necroptosis signalling. FEBS J 282: 19-31, 2015.

41. Bray K, Mathew R, Lau A, Kamphorst JJ, Fan J, Chen J, Chen HY, Ghavami A, Stein M, DiPaola RS, et al: Autophagy suppresses RIP kinase-dependent necrosis enabling survival to mTOR inhibition. PLoS One 7: e41831, 2012. 